\title{
Screening for colorectal cancer after pancreatoduodenectomy for ampullary cancer
}

\author{
Pim B. Olthof ${ }^{\text {a, b, * }}$, Jacob L. van Dam ${ }^{\text {b }}$, Jesse V. Groen ${ }^{c}$, Charlotte Oude Ophuis ${ }^{\mathrm{d}}$, \\ Erwin van der Harst ${ }^{\mathrm{d}}$, Peter Paul Coene ${ }^{\mathrm{d}}$, Bert A. Bonsing ${ }^{\mathrm{c}}$, J. Sven D. Mieog ${ }^{\mathrm{c}}$, \\ Hermien Hartog ${ }^{\mathrm{b}}$, Casper van Eijck ${ }^{\mathrm{b}}$, Bas Groot Koerkamp ${ }^{\mathrm{b}}$, \\ Daphne Roos ${ }^{a}$, on behalf of Southwest Pancreatic Cancer Care (SWPCC) \\ a Department of Surgery, Reinier de Graaf Gasthuis, Delft, the Netherlands \\ ${ }^{\mathrm{b}}$ Department of Surgery, Erasmus MC, Rotterdam, the Netherlands \\ ' Department of Surgery, Leiden University Medical Center, Leiden, the Netherlands \\ d Department of Surgery, Maasstad Ziekenhuis, Rotterdam, the Netherlands
}

\section{A R T I C L E I N F O}

\section{Article history:}

Received 11 July 2019

Received in revised form

11 August 2019

Accepted 10 October 2019

Available online $\mathrm{xxx}$

\section{Keywords:}

Ampullary cancer

Colorectal cancer

Pancreaticoduodenectomy

\begin{abstract}
A B S T R A C T
Background: In some Dutch pancreatic surgery centers, patients who underwent pancreatoduodenectomy (PD) for ampullary cancer undergo surveillance for colorectal cancer (CRC), since an association is suggested in contemporary literature. This study aimed to examine the CRC incidence after PD for ampullary cancer in four pancreatic surgery centers and a Dutch nationwide cohort.

Methods: All patients who underwent resection of ampullary cancer from 2005 through 2017 at four centers were included. All colonoscopies and CRC diagnoses in these patients were recorded. In addition all PDs for ampullary cancer in the Dutch Pathology Registry (2000-2017) were recorded along with the CRC diagnoses and compared with an age, sex, and year-matched cohort.

Results: Out of 287 included patients by the four centers, $11 \%$ underwent a colonoscopy within one year after PD. Eight (2.7\%) were diagnosed with CRC before PD and two (0.7\%), at 14 and 72 months after PD. In the nationwide cohort comparison, the CRC incidence was similar before $(2.6 \%$ versus $1.9 \%, \mathrm{P}=0.424)$ and after surgery $(2.1 \%$ versus $3.1 \%, \mathrm{P}=0.237)$. Within one year after $\mathrm{PD}$, the incidence was $0.3 \%$ compared to $0.6 \%$ in the matched controls $(\mathrm{P}=0.726)$.

Conclusions: The current study could not find an increased risk of CRC in patients with resected ampullary cancer. Therefore, there is insufficient justification to screen for CRC in patients with resected ampullary cancer.
\end{abstract}

๑) 2019 Published by Elsevier Ltd.

\section{Introduction}

Ampullary cancer is a rare malignancy that accounts for approximately $0.2 \%$ of all gastrointestinal cancers [1]. Due to its origin distal to the bile and pancreatic duct confluence at the duodenal outflow, the onset of symptoms (e.g. biliary obstruction) is early compared to most other periampullary and pancreatic tumors. As a consequence, resection rates of ampullary cancers reach up to $92 \%$ [2] and the reported 5-year survival rates range from 38 to $67 \%[3-5]$.

\footnotetext{
* Corresponding author. Department of Surgery Erasmus Medical Center, Doctor Molewaterplein 40, 3015, GD, Rotterdam, the Netherlands.

E-mail address: p.olthof@erasmusmc.nl (P.B. Olthof).
}

Several reports have suggested an increased incidence of colorectal polyps and malignancies in patients with ampullary cancer. Ampullary cancer in some patients is associated with hereditary colorectal cancer (CRC) syndromes, such as hereditary nonpolyposis colorectal cancer (HNPCC) [6] and familial adenomatous polyposis (FAP) [7-9]. However, in ampullary cancer patients without these syndromes, the CRC incidence is also reported to be higher than in age-adjusted control groups [10,11].

This association has led to colorectal surveillance in patients who underwent pancreatoduodenectomy (PD) for ampullary cancer in some pancreatic cancer centers in the Netherlands. The yield of routine colonoscopy within one year after surgery is subject of debate.

This study aimed to investigate the incidence of CRC at baseline 
and during follow up after PD for ampullary cancer in four pancreatic surgery centers. Subsequently, the incidence of CRC was investigated in the Dutch nationwide pathology database.

\section{Methods}

All consecutive patients who underwent PD from January 2005 through December 2017 at four Dutch pancreatic surgery centers with a diagnosis of ampullary cancer were included. All patients were selected from prospectively maintained databases at the individual centers based on the postoperative final pathology reports. Patients with benign or premalignant (i.e. dysplasia without invasion) disease of the ampulla and patients diagnosed with either FAP or HNPCC were excluded. All additional data was retrieved from the electronic medical records. The need of ethical approval and the need for individual informed consent was waived by the institutional medical ethics committee.

\section{Primary outcome}

All colonoscopy procedures in the patient cohort both before and after PD were reviewed and scored. The procedural data and number of found and biopsied colorectal polyps were scored, and the outcomes at pathology were recorded. In addition, both previous diagnoses of CRC in the patient cohort before surgery were scored, as were all diagnoses of CRC during follow up after PD.

\section{Variables}

All postoperative complications within 30 days after surgery were scored and graded according the classification proposed by Dindo et al., with major complications defined as grade III or higher [12]. The incidence of postoperative pancreatic fistula [13], biliary leakage, was scored and graded according to the respective ISGPS definitions. Readmissionswithin 30 days after surgery and 90mortality were scored as other outcome parameters.

Survival was defined as the time between the PD and date of death or last follow up. Recurrence-free survival was defined as time from PD to the diagnoses of recurrence, usually on imaging studies. In order to identify subgroups of patients with inferior prognosis in which the relevance of CRC screening would be less relevant, prognostic variables such as TNM stage (7th edition), differentiation grade, and resection margin were recorded.

\section{Dutch Pathology Registry}

Due to the centralization of pancreatic surgery in the Netherlands, patients are usually referred for surgery to a pancreatic surgery center. Follow-up can be conducted either at the pancreatic surgery center or at the referral center. Colonoscopies performed outside the pancreatic surgery center might have been missed. Therefore, all patients who underwent PD between 2000 and 2017 with ampullary cancer as pathology diagnosis were extracted from the nationwide network and registry of histo- and cytopathology in the Netherlands (PALGA) [14]. All diagnoses of colorectal polyps and malignancies were linked to these patients. Using this strategy all patients nationwide were identified along with all their colorectal pathology diagnosis conducted in any hospital nationwide.

A matched control group was selected from patient who underwent diagnostic excision of a mole. These control patients were matched based on a similar 5-year age category at time of the procedure, sex, and the year of the procedure (i.e., diagnostic excision of a mole or pancreaticoduodenectomy). All colorectal diagnoses before and after the mole excision were identified and scored.

\section{Statistical analysis}

Continuous data were presented as median with inter-quartilerange (IQR), except for survival durations which were presented as median with $95 \%$ confidence intervals (95\%CI). Categorical data were presented as number (percentages) and differences were tested using chi-square of Fisher's exact tests. Survival curves were generated according to the Kaplan Meier method and curves were compared using log-rank tests. Length of follow-up was estimated using the reverse Kaplan Meier method. All statistical analyses were performed using SPSS (Version 23.0, IBM, Chicago, IL) and figures were generated with Graphpad Prism (Graphpad inc, La Jolla, CA).

\section{Results}

\section{Four center cohort}

In the study period, 289 patients underwent PD for ampullary cancer. Two patients diagnosed with a hereditary polyposis syndrome were excluded and the remaining 287 patients were included in the analyses. Baseline characteristics are provided in Table 1 and postoperative outcomes in Table 2. Median (95\% CI) follow up was 83 months (64-104), with a median (95\% CI) overall survival of 35 (25-45) months and 5-year survival was $40 \%$.

Eight patients (2.7\%) were diagnosed with CRC before PD, seven had colon cancer and the remaining two had rectal cancer. All underwent curative resection with a median (range) of 39 (22-70) months before PD and a single patient underwent additional curative resection of colorectal liver metastases 74 months before ampullary cancer resection.

Thirty-three patients (11\%) underwent a colonoscopy within one year after PD all with surveillance as indication. The proportion of patients who underwent a colonoscopy within one year ranged from $3 \%$ to $15 \%$ across the four included centers $(P=0.064)$. In nine of these procedures, no polyps were found and 22 procedures resulted in the resection or biopsy of hyperplastic polyps or polyps with low grade dysplasia. No CRC was diagnosed within one year of PD. During subsequent follow-up of these 33 patients none were diagnosed with CRC.

During further follow-up an additional 14 patients underwent a colonoscopy. Two of these $(0.7 \%)$ patients were diagnoses with CRC (Fig. 1). The first patient was diagnosed with rectal cancer 14 months after PD and underwent curative resection of a T1N0 tumor, the other patient had a right-sided colonic tumor 72 months after PD and was diagnosed with metastatic lesions from the ampullary

Table 1

Baseline characteristics.

\begin{tabular}{ll}
\hline & $\mathrm{N}=287$ \\
\hline $\begin{array}{l}\text { Age, years, median (IQR) } \\
\text { Male gender, } n(\%)\end{array}$ & $68(60-74)$ \\
Center & $166(58)$ \\
EMC & \\
LUMC & $145(51)$ \\
RDGG & $65(23)$ \\
MZH & $39(14)$ \\
ASA score, n (\%) & $38(13)$ \\
III-IV & \\
Pyloris preserving PD, n (\%) & $42(15)$ \\
Minimally invasive approach, $n(\%)$ & $203(71)$ \\
Laparoscopic & $2(1)$ \\
Robotic & $3(1)$ \\
\hline
\end{tabular}


Table 2

Postoperative outcomes.

\begin{tabular}{ll}
\hline & $\mathrm{N}=287$ \\
\hline Highest complication grade, $\boldsymbol{n}(\%)$ & $184(64)$ \\
O-II & $44(15)$ \\
IIIA & $7(2)$ \\
IIIB & $33(11)$ \\
IVA & $3(1)$ \\
IVB & $16(6)$ \\
V (30-day mortality & \\
Postoperative pancreatic fistula, $n$ (\%) & $62(22)$ \\
Grade B/C & \\
Biliary leakage, $n(\%)$ & $19(7)$ \\
Grade B/C & $29(10)$ \\
Readmission rate, $n(\%)$ & $18(6)$ \\
$\mathbf{9 0 - d a y ~ m o r t a l i t y , ~} n(\%)$ & \\
T-stage, $\mathbf{n}(\%)(\mathbf{n}=\mathbf{2 8 2})$ & $46(16)$ \\
T1 & $97(34)$ \\
T2 & $99(35)$ \\
T3 & $40(14)$ \\
T4 & $140(49)$ \\
Node positive disease, $\mathbf{n}(\%)(\mathbf{n}=\mathbf{2 8 6})$ & $20(15-30)$ \\
Tumor size, mm, median $(\mathbf{I Q R})(\mathbf{n}=\mathbf{2 8 2})$ & \\
Differentiation, $\mathbf{n}(\%)(\mathbf{n}=\mathbf{2 6 5})$ & $68(26)$ \\
Well & $130(49)$ \\
Moderate & $67(25)$ \\
Poor & $257(90)$ \\
R0 resection rate, $n(\%)$ & \\
\hline
\end{tabular}

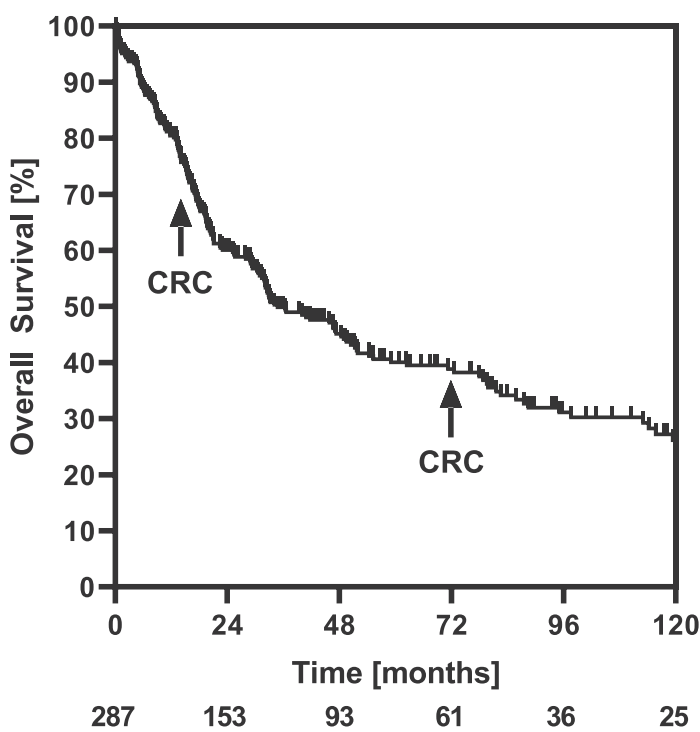

Fig. 1. Overall survival in the 287 patients who underwent pancreatoduodenectomy for ampullary cancer. The two diagnoses of colorectal cancer during follow up are indicated by the arrows. Below the graph are the number of patients at risk.

cancer during staging.

Considering a median (95\% CI) follow-up of 83 (64-104) months after PD in the 287 patients, the person years follow-up was 1044 years. Consequently, there was 1 CRC event per 522 person years of follow-up.

\section{Dutch Pathology Registry cohort}

From the Dutch Pathology Registry, 901 patients were identified who underwent PD for ampullary cancer between 2000 and 2017, which includes the four-center cohort of 287 patients mentioned above. Twenty-three (2.6\%) out of the 901 patients were diagnosed with CRC before PD, all of whom underwent curative resection. One of these patients was treated for a primary colon cancer twice before PD. Sixty-six patients (7\%) underwent a colonoscopy with a biopsy for pathology analysis within one year after PD. In three of these patients, CRC was diagnosed. For one of these patients, this was a second primary colon cancer. During subsequent follow-up, an additional 66 (7\%) patients underwent colonoscopy with a biopsy. In sixteen of these patients, CRC was diagnosed and one patient had a colonic lesion that turned out to be a ampullary cancer metastasis. This translates into a postoperative CRC incidence of $2.1 \%$ (19/901). The diagnosis of adenocarcinoma was made at a median follow-up of $3(2-6)$ years after PD.

The results from the ampularry cancer patients were compared to 901 control patients who underwent diagnostic excision of a mole, matched for age, sex, and the year of surgery (Table 3 ). In the matched cohort, 17 (1.9\%) patients were diagnosed with CRC before the diagnostic excision $(P=0.424)$. Five patients were diagnosed with CRC within one year after surgery $(P=0.726)$. In the matched cohort, the postoperative CRC incidence was 3.1\% (28/901) diagnosed at a median follow-up of $4(2-8)$ years after mole excision $(\mathrm{P}=0.237)$.Overall, 39 (4.7\%) ampullary cancer patients were diagnosed with CRC either before or after surgery compared to 45 (5.0\%) in the control group $(\mathrm{P}=0.826)$.

\section{Discussion}

This study reports on 287 patients who underwent PD for ampullary cancer. Thirty-three (11\%) patients underwent a colonoscopy either the year of or year after ampullary cancer surgery and none of these procedures CRC was diagnosed. Two (1\%) patients were diagnosed with CRC, 14 and 72 months after PD. At a national level, 901 patients underwent PD for ampullary cancer over an 18 year period. The incidence of CRC after PD was similar (2.1\%) to a matched control group (3.1\%, $\mathrm{P}=0.237)$. Within one year after surgery, the CRC incidence was $0.3 \%$ and $0.6 \%(P=0.0726)$, respectively.

Patients diagnosed with familiar adenomatous polyposis syndrome have an increased lifetime risk of $3-4 \%$ for duodenal cancer including ampullary cancer [15]. Duodenal malignancies are the main cause of cancer-related death in these patients, which is why these patients undergo routine surveillance for duodenal tumors $[8,16]$. Hereditary non polyposis CRC is also associated with an increased risk of ampullary cancer [6]. To what extent sporadic ampullary cancer is associated with colorectal polyps and malignancies is less well defined.

Several case reports and case series have reported on the incidence of ampullary cancer in association with other tumors, particularly CRC [17-20]. More recent studies showed similarities in pathogenesis between ampullary and CRC, including similar genetic alterations $[20,21]$. A report including 7 cases of ampullary cancer and 19 ampullary adenomas found colorectal polyps in $23 \%$ of patients compared to $26 \%$ in an age-matched control group [10]. However, there were two cases of CRC in the ampullary group and none in the control group, therefore the authors suggested colorectal screening in the work-up for ampullary neoplasms. A larger report identified 2043 patients with pathology confirmed ampullary cancer, of which 30 (1.4\%) developed CRC during follow up as opposed to 14 expected cases based on age-related incidence, resulting in a two-fold higher incidence [11].

Although these studies report on an increased incidence of CRC in ampullary cancer patients, the clinical relevance of the diagnoses and colorectal screening is unknown. The present study found 19 cases of CRC after resection of ampullary cancer in 901 patients, which translates into a $2.1 \%$ incidence, which was slightly higher compared to the $1.4 \%$ in the study by Das et al. In the four-center cohort, the CRC incidence after PD was one per 522 person follow-up years, compared to 189 after PD and 405 in the control 
Table 3

Comparison of the CRC after PD for ampullary cancer and the matched control group from PALGA: Dutch Pathology Registry.

\begin{tabular}{|c|c|c|c|}
\hline & Ampullary cancer patients & Matched controls & P-value \\
\hline \multicolumn{4}{|l|}{ CRC diagnosis, $n(\%)$} \\
\hline Before surgery & $23(2.6)$ & $17(1.9)$ & 0.424 \\
\hline Within one year of surgery & $3(0.3)$ & $5(0.6)$ & 0.726 \\
\hline After surgery & $19(2.1)$ & $28(3.1)$ & 0.237 \\
\hline Total & $42(4.7 \%)$ & $45(5.0)$ & 0.826 \\
\hline
\end{tabular}

group in the Das et al. report, which included both resected and unresected ampullary cancer patients, compared to resected patients in the present study. Since the prognosis of unresected ampullary cancer is dismal with no long-term survival [22], the follow-up of the present cohort is likely longer and since patients have to be alive to be diagnosed with CRC this has likely influenced the differences.

The CRC incidence in the age-matched control group was 3.1\%, which was slightly higher but non-significant compared to the ampullary cancer group. This could be to the likely inferior prognosis of the ampullary cancer patients compared to the control who underwent diagnostic excision of a mole. Due to the set-up of the PALGA registry that includes only pathology diagnoses, no median follow-up duration was available. Nevertheless, the $2.1 \%$ in the intervention group compared to the $3.1 \%$ incidence in the control group does not support a clinically relevant increased risk of CRC after PD for ampullary cancer, especially since the overall incidence of CRC before or after the reference procedure was similar both cohorts.

Only three patients out of 901 were diagnosed with CRC within one year after PD. This suggests a standard screening of CRC in these patients does not outweigh the associated adverse effects of colonoscopy such as bleeding and perforation and the associated costs $[23,24]$. Especially since the incidence in the control group was 5 diagnoses out of the 901 patients who underwent diagnostic excision of a mole, in which no physician would consider screening for colorectal polyps.

Median overall survival was 36 (27-46) months after resection and 5 -year overall survival was $40 \%$. Survival is strongly influenced by positive lymph nodes, resection margin, tumor grade, as well as lymphovascular, and perineural invasion $[25,26]$. In the presence of any of these factors, survival is poor. Conversely, in patients with a radical resection of a well differentiated tumor with negative lymph nodes might have long term survival. Considering the survival rates in the presence of one of more of these prognostic factors, all efforts to screen for colorectal polyps will likely not impact the outcomes for these patients.

This report has several limitations. The main limitation is the retrospective study design which, considering the low amount of patients that underwent colonoscopy, is subject to verification bias. However, the nationwide pathology data is of high quality due to the all-inclusive nationwide data which included diagnoses of colorectal polyps of these patients in any hospital and revealed similar results. Finally, since a colonoscopy and/or biopsy had to be performed in order to include the colorectal diagnoses in the report, it cannot be excluded that some patients with (asymptomatic) CRC were missed. Furthermore, some patients might have been part of the colorectal cancer screening program using stool sampling implemented in the Netherlands for all inhabitants ages 55 to 75 starting January 2014.

In conclusion, $2.1 \%$ of patients who undergo PD for ampullary cancer are diagnosed with CRC during follow-up. Since only 3 patients were diagnosed within one year of surgery, compared to 5 patients after diagnostic mole excision, standard perioperative screening of CRC in these patients is likely irrelevant. Especially in patients with advanced tumor stage or tumor-related characteristics associated with poor prognosis, the relevance of (asymptomatic) CRC is questionable due to the limited prognosis. The current study could not find an increased risk of colorectal malignancies in patients with resected ampullary cancer. Therefore, there is insufficient justification to screen for colorectal polyps and malignancies in patients with resected ampullary cancer.

\section{Additional information}

The need for ethical approval and individual informed consent was waived by the Medical Ethics committee of Southwest Holland. The Study was performed in accordance with the Declaration of Helsinki. The data for this study is available upon request. None of the authors report any conflicts of interest and no funding was received for the current study.

\section{Declaration of competing interest}

The authors report no conflicts of interest.

\section{References}

[1] Jemal A, Siegel R, Ward E, Hao Y, Xu J, Murray T, et al. Cancer statistics. CA Cancer J Clin 2008 2008;58:71-96.

[2] Bettschart V, Rahman MQ, Engelken FJ, Nadhavan KK, Parks RW, Garden OJ Presentation, treatment and outcome in patients with ampullary tumours. $\mathrm{Br}$ J Surg 2004;91:1600-7.

[3] Todoroki T, Koike N, Morishita Y, Kawamoto T, Ohkohchi N, Shoda J, et al Patterns and predictors of failure after curative resections of carcinoma of the ampulla of Vater. Ann Surg Oncol 2003;10:1176-83.

[4] Talamini MA, Moesinger RC, Pitt HA, Sohn TA, Hruban RH, Lillemoe KD, et al. Adenocarcinoma of the ampulla of Vater. A 28-year experience. Ann Surg 1997:225:590-9. discussion 9-600.

[5] Duffy JP, Hines OJ, Liu JH, Ko CY, Cortine G, Isacoff WH, et al. Improved survival for adenocarcinoma of the ampulla of Vater: fifty-five consecutive resections. Arch Surg 2003;138:941-8. discussion 8-50.

[6] Bansidhar B]. Extracolonic manifestations of lynch syndrome. Clin Colon Rectal Surg 2012;25:103-10.

[7] Sulbaran M, Campos FG, Ribeiro U, Kishi HS, Sakai P, de Moura EGH, et al. Risk factors for advanced duodenal and ampullary adenomatosis in familia adenomatous polyposis: a prospective, single-center study. Endosc Int Open 2018;6:E531-40.

[8] van Heumen BW, Nieuwenhuis MH, van Goor H, Mathus-Vliegen LE, Dekker E Gouma DJ, et al. Surgical management for advanced duodenal adenomatosis and duodenal cancer in Dutch patients with familial adenomatous polyposis: a nationwide retrospective cohort study. Surgery 2012;151:681-90.

[9] Kashiwagi H, Spigelman AD, Debinski HS, Talbot IC, Philips RK. Surveillance of ampullary adenomas in familial adenomatous polyposis. Lancet 1994;344: 1582.

[10] Schneider AR, Seifert H, Trojan J, Stein J, Hoepffner NM. Frequency of colorectal polyps in patients with sporadic adenomas or adenocarcinomas of the papilla of vater-an age-matched, controlled study. Z Gastroenterol 2005;43: $1123-7$.

[11] Das A, Neugut AI, Cooper GS, Chak A. Association of ampullary and colorectal malignancies. Cancer 2004;100:524-30.

[12] Dindo D, Demartines N, Clavien PA. Classification of surgical complications: a new proposal with evaluation in a cohort of 6336 patients and results of survey, Ann Surg 2004:240:205-13.

[13] Bassi C, Marchegiani G, Dervenis C, Sarr M, Abu Hilal M, Adham M, et al. The 2016 update of the International Study Group (ISGPS) definition and grading of postoperative pancreatic fistula: 11 Years after. Surgery 2017:161:584-91.

[14] Casparie M, Tiebosch AT, Burger G, Blauwgeers H, van de Pol A, van Krieken JH, et al. Pathology databanking and biobanking in The Netherlands, a central role for PALGA, the nationwide histopathology and cytopathology data 
network and archive. Cell Oncol 2007;29:19-24.

[15] Vasen HF, Bulow S, Myrhoj T, Mathus-Vliegen L, Griffioen G, Buskens E, et al. Decision analysis in the management of duodenal adenomatosis in familial adenomatous polyposis. Gut 1997;40:716-9.

[16] Offerhaus GJ, Giardiello FM, Krush AJ, Booker SV, Tersmette AC, Kelley NC, et al. The risk of upper gastrointestinal cancer in familial adenomatous polyposis. Gastroenterology 1992;102:1980-2.

[17] Karayiannakis AJ, Kakolyris S, Kouklakis G, Xenidis N, Bolanaki H, Tsalikidis C et al. Synchronous carcinoma of the ampulla of vater and colon cancer. Case Rep Gastroenterol 2011;5:301-7.

[18] Rajalingam R, Javed A, Gondal R, Arora A, Nag HH, Agarwal AK. Non-familial double malignancy of the colon and ampulla of Vater: a case report and review of literature. Saudi J Gastroenterol 2012;18:143-5.

[19] Eriguchi N, Aoyagi S, Tamae T, Nishimura K, Hamada S, Kawabata M, et al. Carcinoma of the ampulla of Vater associated with other organ malignancies. Kurume Med J 2001:48:255-9.

[20] Fischer HP, Zhou H. Pathogenesis of carcinoma of the papilla of Vater. J Hepatobiliary Pancreat Surg 2004;11:301-9.
[21] Howe JR, Klimstra DS, Cordon-Cardo C, Paty PB, Park PY, Brennan MF. K-ras mutation in adenomas and carcinomas of the ampulla of vater. Clin Cancer Res 1997:3:129-33.

[22] Richmond IL, Wilson CB. Pituitary adenomas in childhood and adolescence. Neurosurg 1978;49:163-8.

[23] Siriwardana RC, Chan SC, Chok KS, Lo CM, Fan ST. Effects of the liver volume and donor steatosis on errors in the estimated standard liver volume. Liver Transplant 2011;17:1437-42.

[24] Zauber AG. Cost-effectiveness of colonoscopy. Gastrointest Endosc Clin N Am 2010;20:751-70.

[25] Allema JH, Reinders ME, van Gulik TM, van Leeuwen DJ, Verbeek PC, de Wit LT, et al. Results of pancreaticoduodenectomy for ampullary carcinoma and analysis of prognostic factors for survival. Surgery 1995;117:247-53.

[26] Moekotte AL, Lof S, van Roessel S, Fontana M, Dreyer S, Shablak A, et al. Histopathologic predictors of survival and recurrence in resected ampullary adenocarcinoma: international multicenter cohort study. Ann Surg 2019. In press. 